\title{
Transient gamma-ray emission from Cygnus X-3
}

\section{Anabella T. Araudo}

Instituto Argentino de Radioastronomía, C.C.5, (1894) Villa Elisa, Buenos Aires, Argentina. Facultad de Ciencias Astronómicas y Geofísicas, Universidad Nacional de La Plata, Paseo del Bosque, 1900 La Plata, Argentina.

E-mail: aaraudodfcaglp.unlp.edu.ar

\section{Valentí Bosch-Ramon}

Dublin Institute for Advanced Studies, 31 Fitzwilliam Place, Dublin 2.

E-mail: valenti@cp.dias.ie

\section{Gustavo E. Romero}

Instituto Argentino de Radioastronomía, C.C.5, (1894) Villa Elisa, Buenos Aires, Argentina.

Facultad de Ciencias Astronómicas y Geofísicas, Universidad Nacional de La Plata, Paseo del Bosque, 1900 La Plata, Argentina.

E-mail: romero@fcaglp.unlp.edu.ar

The high-mass microquasar Cygnus X-3 has been recently detected in a flaring state by the gamma-ray satellites Fermi and Agile. In the present contribution, we study the high-energy emission from Cygnus X-3 through a model based on the interaction of clumps from the WolfRayet wind with the jet. The clumps inside the jet act as obstacles in which shocks are formed leading to particle acceleration and non-thermal emission. We model the high energy emission produced by the interaction of one clump with the jet and briefly discus the possibility of many clumps interacting with the jet. From the characteristics of the considered scenario, the produced emission could be flare-like due to discontinuous clump penetration, with the GeV long-term activity explained by changes in the wind properties.

25th Texas Symposium on Relativistic Astrophysics - TEXAS 2010

December 06-10, 2010

Heidelberg, Germany 

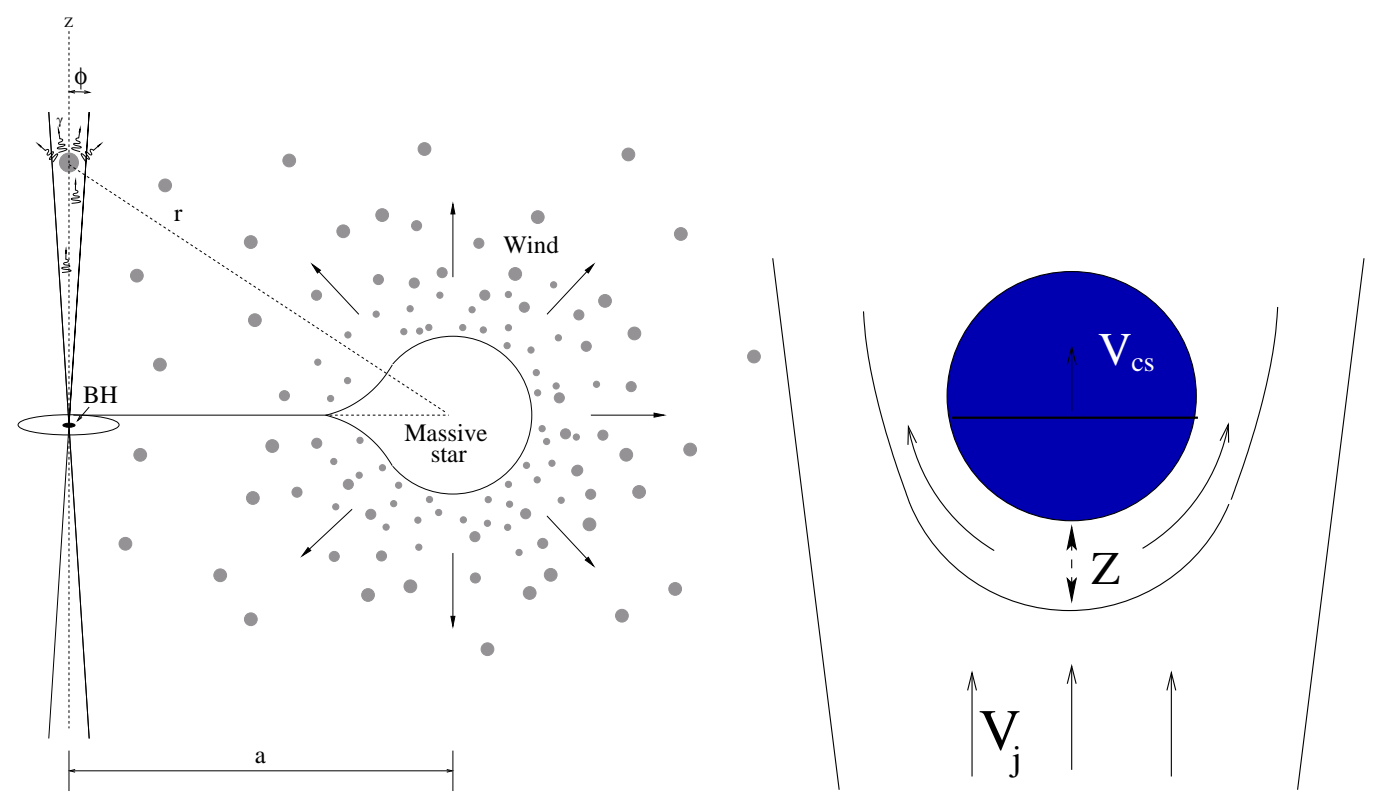

Figure 1: Left: Sketch of a HMMQ. Right: Sketch of the interaction of one clump with the jet.

\section{Introduction}

The mass loss in massive stars is thought to take place via supersonic inhomogeneous winds. Considerable observational evidence supports the idea that the wind structure is clumpy [1], although the properties of clumps are not well-known as a consequence of the very high spatial resolution necessary for clump detection. Some massive stars are accompanied by a compact object and present transfer of matter to the latter, forming an accretion disc. When bipolar relativistic outflows are formed, these sources are called high-mass microquasars (HMMQs). In Figure 1 (left), a sketch of a HMMQ is shown.

Cygnus X-3 is a HMMQ composed by a Wolf-Rayet (WR) star and a compact object, both being separated a distance $a \sim 3 \times 10^{11} \mathrm{~cm}$. The nature of the compact object has not been determined yet, but recent studies suggest that it may be a black hole (e.g. [2]). Located at a distance $\sim 7-9 \mathrm{kpc}$, the system has been recently detected in a recurrent flaring state at high-energy (HE) gamma rays [3, 4]. The observed emission spectrum is a power-law with index $\sim 1.7$ and luminosity $\gtrsim 10^{36} \mathrm{erg} \mathrm{s}^{-1}$. The typical duration of the flares was $\sim 2$ days [4].

In this contribution, we present a model to explain the HE emission detected in Cygnus X-3 based on the interaction between its jets and wind inhomogeneities [5]. Some clumps can eventually penetrate in the jet, leading to transient non-thermal activity that may release a significant fraction of the jet kinetic luminosity, $L_{\mathrm{j}}$, in the form of synchrotron, inverse Compton (IC), and proton-proton $(p p)$ emission.

\section{Jet-clump interaction in Cygnus X-3}

In order to study the physical processes of the jet-clump interaction, we consider the collision of a single clump with the jet, as is shown in Figure 1 (right). A huge density contrast $\chi \equiv \rho_{\mathrm{c}} / \rho_{\mathrm{j}}$ 
(where $\rho_{\mathrm{c}}$ and $\rho_{\mathrm{j}}$ are the clump and jet densities, respectively) allows the clump to fully cross the boundary of the jet and penetrate into it. In the context of this work, thermal conduction, magnetic fields and gravitational forces are not dynamically relevant for the jet-clump interaction and have been neglected.

We consider that a clump with size $R_{\mathrm{c}}^{0}=10^{10} \mathrm{~cm}\left(\lesssim 0.1 R_{\star}\right)$ reaches one of the HMMQ jets moving at the terminal wind velocity $v_{\infty}=2500 \mathrm{~km} \mathrm{~s}^{-1}$. At a distance $r=\sqrt{z^{2}+a^{2}}$ from the star, the clump density can be estimated through $\rho_{\mathrm{c}}=\rho_{\mathrm{w}} / f$, where $f<1$ is the filling factor of the clumpy wind, and $\rho_{\mathrm{w}} \sim \dot{M} /\left(4 \pi r^{2} v_{\infty}\right)$ is the wind density, where $\dot{M} \sim 10^{-5} \mathrm{M}_{\odot} \mathrm{yr}^{-1}$ is the typical mass loss rate of WR stars. We assume that the jet has a velocity $v_{\mathrm{j}} \sim c / 3$ (i.e. Lorentz factor $\Gamma \sim 1.06)$ and a kinetic luminosity $L_{\mathrm{j}}=10^{38} \mathrm{erg} \mathrm{s}^{-1}$. We fix the relation between the height (z) and the width $\left(R_{\mathrm{j}}\right)$ of the jet as $R_{\mathrm{j}}=0.1 z$.

The penetration time of the clump into the jet is determined by $t_{\mathrm{c}} \sim 2 R_{\mathrm{c}}^{0} / v_{\mathrm{c}} \sim 100 \mathrm{~s}$, where the clump velocity is $v_{\mathrm{c}}=v_{\infty}$. As a consequence of the interaction of the jet material with the clump, two shocks form. One of these shocks propagates back in the jet with a velocity $v_{\mathrm{bs}} \sim v_{\mathrm{j}}$, forming a bow shock (see Figure 1, right). This bow shock reaches the steady state configuration in a time $t_{\mathrm{bs}} \sim Z_{\mathrm{bs}} / v_{\mathrm{bs}}$, with the stagnation point located at a distance $Z_{\mathrm{bs}} \sim R_{\mathrm{c}}^{0} / 5$ from the clump. On the other hand, a shock propagates in the clump at a velocity $v_{\mathrm{sc}} \sim v_{\mathrm{j}} / \sqrt{\chi}$ and, in a time $t_{\mathrm{sc}} \sim 2 R_{\mathrm{c}} / v_{\mathrm{sc}}$, the whole clump is shocked. During the shock passage, the clump is compressed in the direction of the shock velocity, $z$, and when the whole clump is shocked, its size in the $z$-direction is $\sim R_{\mathrm{c}}^{0} / 4$. However, in $t>t_{\mathrm{sc}}$ the clump starts to expand at the sound velocity of the shocked material. At the same time, the acceleration exerted by the jet to the clump increases with $R_{\mathrm{c}}$. Assuming that the expansion is spherical, and neglecting the acceleration exerted by the jet on the clump, we obtain the following expression for the clump size: $R_{\mathrm{c}}(t) \sim R_{\mathrm{c}}^{0} /\left(1-0.4 t / t_{\mathrm{sc}}\right)^{2}$ [6], which gives a characteristic clump expansion time $t_{\exp } \sim 3.5 t_{\mathrm{sc}}$. In Figure 2 (left), the evolution of $R_{\mathrm{c}}$ with time is shown.

As a consequence of the acceleration exerted by the jet on the clump, Rayleigh-Taylor (RT) instabilities can develop in the contact discontinuity. Kelvin-Helmholtz (KH) instability can also grow as a result of the high relative velocity between the jet shocked material and the clump. Numerical simulations show that RT and KH instabilities grow sufficiently to destroy the clump (i.e. up to a wavelength $\sim R_{\mathrm{c}}$ ) in a timescale $t_{\mathrm{RT}} \sim t_{\mathrm{KH}} \sim 5 t_{\mathrm{sc}}$ [7]. If instabilities do not grow fast enough, and clump expansion and acceleration are too slow, the permanence of the clump into the jet will be determined by the passage time of the clump through the jet: $t_{\mathrm{j}} \sim 2 R_{\mathrm{j}} / v_{\mathrm{c}}$. In Figure 2 (right), the main dynamical timescales are plotted.

\subsection{Interaction height}

Considering the previous analysis on the dynamics of the jet-clump interaction, one can determine the minimum interaction height, $z_{\text {int }}^{\min }$, at which one clump can completely penetrate into the jet. Being the lifetime of clumps into the jet $t_{\text {life }} \gtrsim t_{\text {sc }}$, to determine the interaction height $z_{\text {int }}$ we impose that $t_{\mathrm{sc}}>t_{\mathrm{c}}$. This constraint is fulfilled if $z>A \sqrt{z^{2}+a^{2}}$, where

$$
A \sim 0.5\left[\left(\frac{f}{0.01}\right)\left(\frac{v_{\mathrm{j}}}{c / 3}\right)\left(\frac{L_{\mathrm{j}}}{10^{38} \mathrm{erg} / \mathrm{s}}\right)\right]^{1 / 2}\left[\left(\frac{\Gamma_{\mathrm{j}}-1}{0.06}\right)\left(\frac{\dot{M}}{10^{-5} M_{\odot} / \mathrm{yr}}\right)\left(\frac{v_{\infty}}{2500 \mathrm{~km} / \mathrm{s}}\right)\right]^{-1 / 2} .
$$



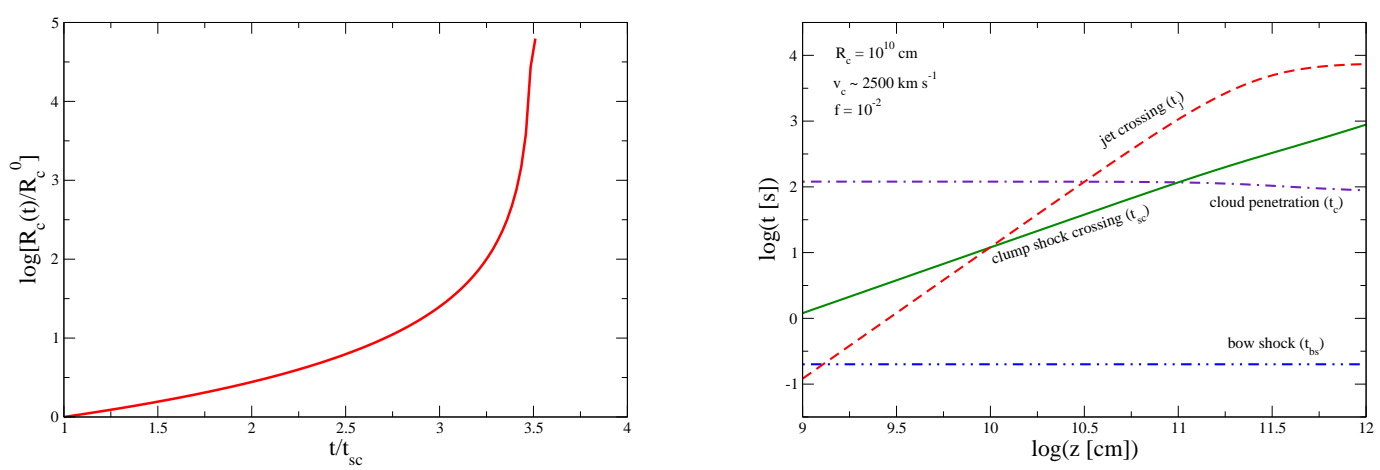

Figure 2: Left: Clump spherical expansion. Right: Dynamical timescales corresponding to the interaction of one clump of $R_{\mathrm{c}}^{0}=10^{10} \mathrm{~cm}$ with the jet.

If, for a certain election of clump and jet parameters, $A$ results larger than 1 , the constrain $t_{\mathrm{sc}}>t_{\mathrm{c}}$ is no satisfied for any value of $z$ and clumps will be destroyed by the jet before fully penetrating into it. However, for the parameters chosen in this work, $A \sim 0.5$ and in such a case, the minimum interaction height results $z_{\text {int }}^{\min } \sim A a / \sqrt{1-A^{2}} \sim a / 2$.

\section{Non-thermal processes}

To estimate the non-thermal emission produced by the interaction of one clump with the jet, we fix the interaction height at $z_{\text {int }}=a \sim 2 z_{\text {int }}^{\min }$. At $z_{\text {int }}$, the density of the clump is $\rho_{\mathrm{c}} \sim 10^{-12} \mathrm{gr} \mathrm{cm}^{-3}$ (i.e. the number density is $n_{\mathrm{c}}=\rho_{\mathrm{c}} /\left(4 m_{p}\right) \sim 2 \times 10^{11} \mathrm{~cm}^{-3}$, where $m_{p}$ is the proton mass).

\subsection{Particle acceleration}

In the bow shock, we assume that particles are accelerated up to relativistic energies being injected in the downstream region following a distribution $Q_{e, p} \propto E_{e, p}^{-2}(e$ and $p$ for electrons and protons, respectively) and with a luminosity $L_{\mathrm{nt}}=\eta_{\mathrm{nt}}\left(R_{\mathrm{c}} / R_{\mathrm{j}}\right)^{2} L_{\mathrm{j}}$, where we fix $\eta_{\mathrm{nt}}=0.1$. For the parameters assumed in this work,

$$
L_{\mathrm{nt}} \sim 10^{36}\left(\frac{\eta_{\mathrm{nt}}}{0.1}\right)\left(\frac{R_{\mathrm{c}}^{0}}{10^{10} \mathrm{~cm}}\right)^{2}\left(\frac{z_{\mathrm{int}}}{a=3 \times 10^{11} \mathrm{~cm}}\right)^{-2}\left(\frac{L_{\mathrm{j}}}{10^{38} \mathrm{erg} \mathrm{s}^{-1}}\right) \quad \operatorname{erg~s}^{-1} .
$$

In addition, considering that the magnetic energy density in the bow-shock region, $U_{B}$, is a fraction $\eta_{\mathrm{B}}$ of the non-thermal one, $U_{\mathrm{nt}}$, we obtain a magnetic field $B \sim \sqrt{\eta_{\mathrm{B}}} 2 \times 10^{3}$ G. (Both $\eta_{\mathrm{nt}}$ and $\eta_{\mathrm{B}}$ are free parameters in our work.)

The main radiative losses that affect the evolution of the non-thermal particles are synchrotron radiation, and synchrotron self-Compton (SSC) and external Compton (EC) scattering. For EC cooling we have considered target photons provided by the WR star, that has a luminosity $L_{\star} \sim$ $10^{39} \mathrm{erg} \mathrm{s}^{-1}$ and a temperature $T_{\star} \sim 10^{5} \mathrm{~K}$. The energy density of stellar photons at $z_{\text {int }}$ is $U_{\star} \sim$ $3 \times 10^{4} \mathrm{erg} \mathrm{cm}^{-3}$. At electron energies $E_{e} \gtrsim 25 \mathrm{GeV}$, EC scattering with stellar photons of energy $E_{\mathrm{ph} \star} \sim 3 K T_{\star}$ (where $K$ is the Boltzmann constant) occurs in the Klein-Nishina regime. In addition 

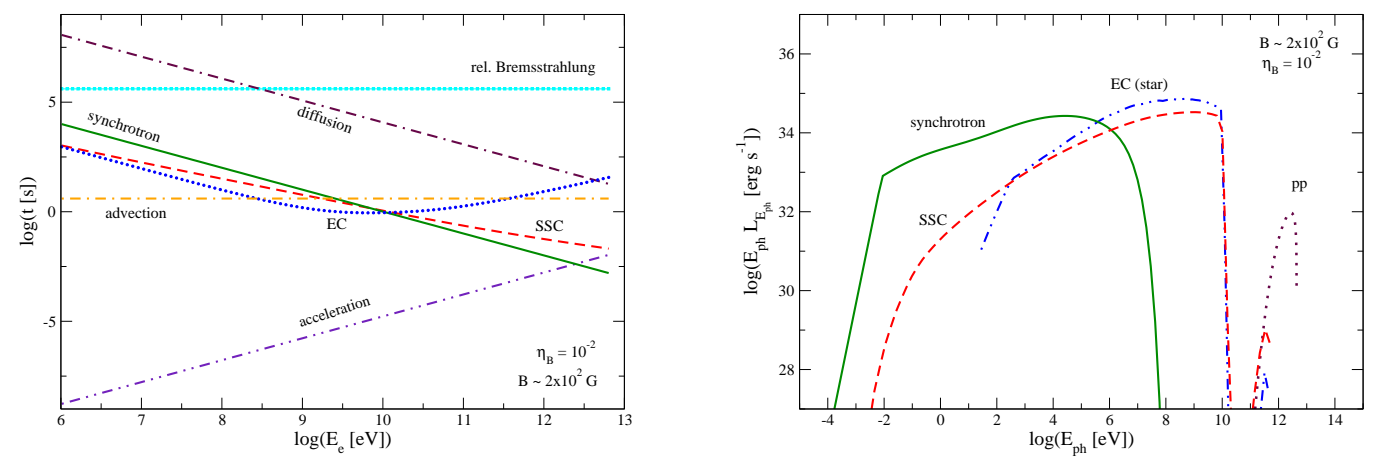

Figure 3: Left: Timescales of the acceleration and lepton cooling losses (synchrotron, SSC, EC and relativistic Bremsstrahlung). Diffusion and advection escape losses are also shown. Right: Spectral energy distribution produced by one clump of $R_{\mathrm{c}}^{0}=10^{10} \mathrm{~cm}$ interacting with the jet at $z_{\text {int }} \sim a$. At energies $E_{\mathrm{ph}} \gtrsim 10 \mathrm{GeV}$, the emission is strongly absorbed. Both (left and right) plots have been produced taking $B \sim 2 \times 10^{2} \mathrm{G}$.

to radiative losses, electrons are advected away from the emitter on a time $t_{\mathrm{adv}} \sim 4 R_{\mathrm{c}} / v_{\mathrm{j}}$. In Figure 3 (left), the electron cooling timescales are plotted together with the acceleration timescale. As seen in the figure, for $\eta_{\mathrm{B}}=10^{-2}$ the maximum energy is determined by synchrotron losses, yielding $E_{e}^{\max } \sim 1 \mathrm{TeV}$. To obtain the distribution $N_{e}$ of relativistic electrons we solve the kinetic equation [8] for a one-zone emitter, taking into account the losses mentioned above, and also the synchrotron photons (as targets for SSC). The steady state is reached on a time $\ll t_{\mathrm{sc}} . N_{e}\left(E_{e}\right)$ has a break at the energy $E_{\mathrm{b}} \sim 0.1 \mathrm{GeV}$ due to electron advection escape.

Protons can lose energy via $p p$ interactions in the bow-shock region, but diffusion losses dominate, constraining the maximum energy to $E_{p}^{\max } \sim 2.5 \times 10^{2} \sqrt{\eta_{\mathrm{B}}} \mathrm{TeV}$ (for $\eta_{\mathrm{nt}}=0.1$ ). The most energetic protons, $E_{p} \gtrsim 0.1 E_{p}^{\max }$, can diffuse up to the clump before escaping through advection, producing gamma rays via $p p$ interactions.

\subsection{High-energy emission}

In the bow-shock region, using standard formulas [9] and $N_{e}$, we estimate the synchrotron, SSC and EC emission; relativistic Bremmstrahlung and $p p$ interactions are negligible there. In the clump, if energetic protons that arrive from the bow shock are not confined, they will escape from the clump in a time $t_{\mathrm{cl}} \sim R_{\mathrm{c}}^{0} / c$ before radiating a significant part of their energy. Considering that the energy distribution of relativistic protons in the clump is $N_{p} \sim Q_{p} t_{\mathrm{cl}}$, we estimate the $p p$ emission following the formulae given in [10]. In Figure 3 (right), we show the spectral energy distribution (SED) composed by the most important radiative processes in the bow-shock region (synchrotron, SSC and EC) and in the clump ( $p p)$. The synchrotron emission is self-absorbed at energies $E_{\mathrm{ph}}<10^{-4} \mathrm{eV}$, and at photon energies $E_{\mathrm{ph}} \gtrsim 10 \mathrm{GeV}$ absorption due to electron-positron pair creation in the star photon field is relevant. The achieved luminosity at energies $\sim 0.1-10 \mathrm{GeV}$ is $L_{\mathrm{EC}} \sim 2 \times 10^{35} \mathrm{erg} \mathrm{s}^{-1}$. Regarding to the $p p$ emission, the estimate obtained in this work is a lower-limit. If the protons were confined in the clump, the radiated emission would be larger.

The SED shown in Figure 3 (right) has been calculated neglecting the expansion of the clump inside the jet (see Figure 2, right). If we consider that after $t_{\mathrm{sc}}$ the cloud expands up to a size much 
larger than $R_{\mathrm{c}}^{0}=10^{10} \mathrm{~cm}$, the available non-thermal luminosity will be significantly larger and, as a consequence, the synchrotron and IC luminosity will be higher. On the other hand, the $p p$ emission produced in the expanded clump will be lower, because the clump density decreases faster than the increase of the clump surface: $n_{\mathrm{c}} / n_{\mathrm{c}}^{0} \propto\left(R_{\mathrm{c}}^{0} / R_{\mathrm{c}}\right)^{3}$.

Finally, note that in Figure 3 (right) we show the result of the interaction of only one clump with the jet, but many clumps could simultaneously interact with the jet. The non-thermal radiation fluxes grow linearly with the number of clumps interacting with the jet, $N_{\mathrm{cj}}$, as long as too many clumps do not disrupt the jet. The corresponding SED will be also higher.

\section{Discussion}

The total luminosity produced by jet-clump interactions depends on the number of clumps inside the jet, and on the size and density of each one. The number of clumps in the jet can be estimated as $N_{\mathrm{cj}} \sim f_{\mathrm{j}} V_{\mathrm{j}} / V_{\mathrm{c}}$, where $f_{\mathrm{j}} \leq f$ is the filling factor of clumps into the jet, and $V_{\mathrm{j}}$ and $V_{\mathrm{c}}$ are the jet and the clump volume, respectively. Considering $V_{\mathrm{j}}$ up to $z \sim 2 a, V_{\mathrm{c}} \sim \pi R_{\mathrm{c}}^{0^{3}}$ (where $R_{\mathrm{c}}^{0}=10^{10} \mathrm{~cm}$ ) and $f_{\mathrm{j}}=f=0.01$, we obtain $N_{\mathrm{cj}} \sim 0.5$ in Cygnus X-3. However, the real number of clumps into the jet will be smaller than the previous estimate, because $f_{\mathrm{j}}<f$ as a consequence of the clump destruction during the penetration process and inside the jet due to RT and $\mathrm{KH}$ instabilities. If $N_{\mathrm{cj}}<1$, the emission produced by the jet-clump interaction will be like a flare with a duration determined by the lifetime of the clumps into the jet.

On the other hand, if we consider clumps with a radius $R_{\mathrm{c}}^{0}=10^{9} \mathrm{~cm}\left(\lesssim 0.01 R_{\star}\right)$, the number of clumps into the jet grows up to $\sim 10^{4}$. Then, the simultaneous interaction of $\sim 10^{4}$ clumps with the jet will produce significantly more luminosity than the emission produced by the interaction of only one clump, that is $\sim 10^{34} \mathrm{erg} \mathrm{s}^{-1}$ at $z_{\text {int }} \sim a$. In that case, the final spectrum will be persistent, with a flickering due to the continuous interactions of many small clumps.

However, the jet can be disrupted by the too small many clumps, in particular if the section of clumps interacting within $\Delta z$ is comparable with the section of the jet: $\Sigma R_{\mathrm{c}}^{2} \sim R_{\mathrm{j}}^{2}$. This will be the case, neglecting clump expansion, for $R_{\mathrm{c}}^{0} \lesssim 10^{9} \mathrm{~cm}$. Note that periods of smaller clump size may lead to jet disruption. Also, changes in the wind could lead to more clumps inside the jet and more effective tapping of the jet luminosity.

\section{References}

[1] S. Owocki, D. Cohen, The Effect of Porosity on X-Ray Emission-Line Profiles from Hot-Star Winds, ApJ, 2006 (648) 565.

[2] C. Shrader, L. Titarchuk1, N. Shaposhnikov New Evidence for a Black Hole in the Compact Binary Cygnus X-3, ApJ, 2010, (718) 488.

[3] A. Abdo. et al. Modulated High-Energy Gamma-Ray Emission from the Microquasar Cygnus X-3, Science, 2009 (326) 1512.

[4] M. Tavani, et al. Extreme particle acceleration in the microquasar CygnusX-3, Nature, 2009 (462) 620.

[5] A. Araudo, V. Bosch-Ramon, G.E. Romero, High-energy emission from jet-clump interactions in microquasars, A\&A, 2009 (503) 673. 
[6] M. Barkov, F. Aharonian, V. Bosch-Ramon, Gamma-ray Flares from Red Giant/Jet Interactions in Active Galactic Nuclei, ApJ 2010 (724) 1517.

[7] R. Klein, C. McKee, P. Colella, On the hydrodynamic interaction of shock waves with interstellar clouds. 1: Nonradiative shocks in small clouds, ApJ 2004 (420) 213.

[8] V. Ginzburg, S. Zyrovatskii, The origin of cosmic rays Gordon \& Breach, New York 1969

[9] G. Blumenthal, R. Gould, Bremsstrahlung, Synchrotron Radiation, and Compton Scattering of High-Energy Electrons Traversing Dilute Gases, RvMP 1970 (42) 237

[10] S. Kelner, F. Aharonian, V. Bugayov, Energy spectra of gamma rays, electrons, and neutrinos produced at proton-proton interactions in the very high energy regime, PhRvD 2006 (74) 4018 